\title{
Authors' reply: Emergence of antibiotic-resistant bacterial strains, methicillin-resistant Staphylococcus aureus and extended spectrum $\beta$-lactamases, and multi-drug resistance are problems similar to global warming
}

\author{
Juliana Pena Porto ${ }^{[1]}$, Deivid Willian da Fonseca Batistão ${ }^{[2]}$ \\ and Rosineide Marques Ribas ${ }^{[2]}$
}

[1]. Faculdade de Medicina, Universidade Federal de Uberlândia, Uberlândia, MG. [2]. Programa de Pós-Graduação em Imunologia e Parasitologia Aplicadas, Universidade de Uberlândia, Uberlândia, MG.

Dear Editor,

The letter entitled Emergence of antibiotic-resistant bacterial strains, methicillin-resistant Staphylococcus aureus and extended spectrum $\beta$-lactamases, and multi-drug resistance are problems similar to global warming by Dr. Pranab Kumar Bhattacharya was very interesting. It highlighted the importance of infections caused by Staphylococcus aureus, which are responsible for the largest epidemics of recent times, particularly in countries such as Brazil.

The epidemiology of $S$. aureus has been the focus of numerous single-center and multicenter surveillance studies; it is dynamic and has changed significantly in recent years ${ }^{1}$. Staphylococcus aureus is considered the most clinically important, gram-positive pathogen in hospitals and is responsible for superficial and invasive infections of the bloodstream, surgical wounds, pneumonia, endocarditis, and osteomyelitis, as mentioned by Dr. Bhattacharya. Most often, these infections are associated with the use of invasive medical devices such as central venous catheters, mechanical ventilation, urinary catheter, artificial valves, pacemakers, and orthopedic implants $^{2}$. Furthermore, treatment of infections caused by $S$. aureus is often complicated by the high prevalence of multidrug-resistant strains, which are a consequence of the indiscriminate and inappropriate use of antimicrobials associated with vertical and horizontal resistance gene transfer ${ }^{1,3}$.

In his paper, Dr. Bhattacharya illustrated the importance of methicillin-resistant Staphylococcus aureus (MRSA) various mechanisms of resistance; of these mechanisms, the presence

\footnotetext{
Address to: Dra. Juliana Pena Porto. FM/UFU. Avenida Pará 1720/Bloco 2U, Campus Umuarama, 38400-902 Uberlândia, MG, Brasil.

Phone: 5534 3218-2236; 5534 8816-7987.

e-mail: ju-nana@hotmail.com

Received 8 August 2014

Accepted 3 December 2014
}

of the genomic island called staphylococcal cassette chromosome mec (SCCmec) is particularly noteworthy. To date, 11 allotypes of $\mathrm{SCCmec}$ in $\mathrm{S}$. aureus have been described on the basis of classes of mec and ccr complex (recombinase cassette chromosome) genes. The SCCmec types I, II, and III are found predominantly in hospital strains (healthcare-associated MRSA), while types IV and $\mathrm{V}$ are found in the community (community-associated MRSA). However, recently, MRSA SCCmec IV was detected in hospitals. This change in the epidemiology of $S$. aureus will impact the therapeutic choices for control of the resulting infections ${ }^{4-6}$. Dr. Bhattacharya presented a few studies that showed very high rates of community-acquired MRSA carriers in India; however, in Brazil, studies show lower rates of carriers of this phenotype. We focus on these high rates with reference to patients with frequent exposure to the hospital environment.

The majority of nosocomial MRSA infections worldwide are caused by 1 of 5 major MRSA belonging to the clonal complex (CC): 5, 8, 22, 30, and 45. Surveillance data collected between 1994 and 2000 identified 5 predominant clones (Brazilian, Iberian, Hungarian, pediatric, and New York/Japan) belonging to these clonal complexes. These clones accounted for almost $70 \%$ of MRSA isolates worldwide. In Brazil, since it was first reported in 1994, the Brazilian clone (ST239-SCCmec type III) has been isolated in hospitals around the country and has already disseminated out of the country. Several genetic variants of the Brazilian clone, which are together known as the Brazilian epidemic clonal complex (BECC), have been identified. The pathogenicity of the $\mathrm{BECC}$ is a result of its multidrug resistance, adhesion ability, invasion of human epithelial cells, production of toxins such as enterotoxins and Panton-Valentine leukocidin, and ability to form biofilms.

Although resistance plays an important role in the prognosis of the likelihood of infection and therapeutic failure, the virulence of MRSA, which results from multiple factors that act simultaneously to evade the host's defenses, has been implicated in nosocomial infections. The capacity of this pathogenic organism is a product of the combined effect of the production 
of virulence factors such as enzymes and toxins; invasiveness and intracellular multiplication, spread in tissues and organs; and particularly, the ability to produce a biofilm ${ }^{6-8}$. Studies on the epidemiology of $S$. aureus including its prevalence, virulence of the strains, antimicrobial resistance, and mechanisms of environmental adaptation such as biofilm formation can contribute effectively to its control.

Dr. Bhattacharya also discussed the role of antibiotics in the emergence and spread of microorganisms such as MRSA: approximately $40 \%$ of hospitalized patients receive antibiotics; however, in approximately $50 \%$ of the cases, the use of antibiotics is unnecessary 9 . Empirical therapy, particularly in intensive care units, is used with a combination of several broadspectrum antibiotics, allowing resistant strains such as MRSA to emerge and spread. These drugs exert a selective pressure on the microorganisms, leading to an ecological impact, and consequently, specific measures are required to control the resulting infection in hospitals. Agencies such as the World Health Organization (WHO) and National Healthcare Safety Network (NHSN) recommend that the antibiotic consumption in hospitals be evaluated regularly. This has facilitated the comparison of the rates of microorganism types and their antibiotic resistance phenotypes, and thereby in establishing useful relationships between the two characteristics ${ }^{10}$.

Regarding the detection of resistance genes described by Dr. Bhattacharya, in our laboratory ${ }^{11}$, we compared multiplex polymerase chain reaction (PCR) assays for the detection of resistance genes. We used classical methods to determine the susceptibility to antibiotics. However, our discrepant findings can be attributed to technical problems. Previous studies have reported that the conventional susceptibility-testing methods, including disk diffusion and agar dilution tests, are sometimes unreliable for the detection of MRSA ${ }^{12}$.

Finally, molecular typing methods such as pulsed-field gel electrophoresis (PFGE) and multilocus sequence typing (MLST) are essential to determine whether epidemiologically related organisms are genetically related; they also assist in outbreak detection, identification of cross-transmission and sources of infection, and monitoring and control of healthcare-related infections. Thus, several epidemiological surveillance systems have incorporated molecular typing techniques in order to include useful information for a better understanding of the pathogenicity and evolutionary trajectory propagation of microorganisms with the potential to spread in the hospital environment.

Pulsed-field gel electrophoresis has been widely used for classification of isolates in clonal lineages and is described in several studies on molecular epidemiology, considering its high discriminatory power, reproducibility and ability to typing PFGE involves MLST of housekeeping genes and sequencing from the allelic profiles that define the types and sequence of respective pathogens' clonal complex of clinical interest ${ }^{1}$.

In conclusion, to plan strategies for the prevention and control infections caused by multidrug-resistant pathogens such as MRSA, it is essential to first understand the spread of these pathogens. Thus, researchers need to analyze and identify indicators and conduct epidemiological surveillance of infections to better understand the epidemiology and pathogenesis of hospital infections caused by $S$. aureus in tertiary care teaching hospitals.

\section{CONFLICT OF INTEREST}

The authors declare that there is no conflict of interest.

\section{REFERENCES}

1. Chambers HF, Deleo FR. Waves of resistance: Staphylococcus aureus in the antibiotic era. Nat Rev Microbiol 2009; 7:629-641.

2. Rosenthal VD, Maki DG, Jamulitrat S, Medeiros EA, Todi SK, Gomez DY, et al. International Nosocomial Infection Control Consortium (INICC) report, data summary for 2003-2008, issued June 2009. Am J Infect Control 2010; 38:95-104.

3. Hiramatsu K, Ito T, Tsubakishita S, Sasaki T, Takeuchi F, Morimoto $\mathrm{Y}$, et al. Genomic basic for methicillin resistance in Staphylococcus aureus. J Infect Chem 2013; 45:117-136.

4. Cruz C, Moreno J, Renzoni A, Hidalgo M, Reyes J, Schrenzel J, et al. Tracking meticillin-resistant Staphylococcus aureus clones in Colombian hospitals over 7 years (1996-2003): emergence of a new dominant clone. Int J Antimicrob Agents 2005; 26:457.

5. Boyle-Vavra S, Ereshefsky B, Wang CC, Daum RS. Successful multiresistant community-associated methicillin-resistant Staphylococcus aureus lineage from Taipei, Taiwan, that carries either the novel staphylococcal chromosome cassette mec (SCCmec) type VT or SCCmec type IV. J Clin Microbiol 2005; 43:4719-4730.

6. Garzoni C, Kelley WL. Staphylococcus aureus: new evidence for intracellular persistence. Trends Microbiol 2009; 17:59-65.

7. Schlievert PM, Strandberg KL, Lin YC, Peterson ML, Leung DY. Secreted virulence factor comparison between methicillin-resistant and methicillin-sensitive Staphylococcus aureus, and its relevance to atopic dermatitis. J Allergy Clin Immunol 2010; 125:39-49.

8. Laabei M, Recker M, Rudkin JK, Aldeljawi M, Gulay Z, Sloan TJ, et al. Predicting the virulence of MRSA from its genome sequence. Genome Res 2014; 24:839-849.

9. Mandell GL, Bennett JE, Dolin R. Mandell, Douglas and Bennett's Principles and Practice of Infectious Diseases. $6^{\text {th }}$ ed. Philadelphia: Elsevier Churchill Livingstone; 2005.

10. Curtis C, Marriott J, Langley C. Development of a prescribing indicator for objective quantification of antibiotic usage in secondary care. J Antimicrobiol Chem 2004; 54:529-533.

11. Ribas RM, Gontijo-Filho PP, Da Costa Darini AL. Conventional versus molecular tests (multiplex PCR and PCR mecA gene) for detection of methicillin-resistant Staphylococcus aureus. Braz J Microbiol 2003; 34:35-37.

12. Martineau F, Picard FJ, Lansac N, Ménard C, Roy PH, Ouellette M, et al. Correlation between the resistance genotype determined by multiplex PCR assays and the antibiotic susceptibility patterns of Staphylococcus aureus and Staphylococcus epidermidis. Antimicrob Agents Chemother 2000; 44:231-238. 